\title{
Evaluación de cepas de Trichoderma spp. en el control de Fusarium spp en ananá.
}

Castillo, Constanza Gi*.; Pérez, Alejandro²; Lovato Echeverria, Alfonso3; Rollhaiser,Ignacio²; Blengini, María C. ${ }^{2}$

${ }^{1}$ Frutas Carayá. www.frutascaraya.com; ${ }^{2}$ Facultad de Ciencias Agropecuarias. Universidad Nacional de Córdoba; ${ }^{3}$ Facultad de Ciencias Agrarias. Universidad Nacional del Nordeste

*E-mail: constanzacastillo@frutascaraya.com

Introducción:El cultivo de ananá (Ananacomosus) en la provincia de Misiones es afectado por las especies del género Fusarium que afectan el $90 \%$ de la superficie cultivada, con pérdidas que rondan el $80 \%$ de la producción. El uso de fungicidas posee una baja eficiencia de control del patógeno, debido a que es un hongo de suelo. Ante esta problemática, surge como alternativa el uso de controladores biológicos. El objetivo del trabajo fue evaluar el comportamiento "in vivo" de Trichoderma spp como antagonista biológico de Fusarium spp .

Materiales y métodos: Se utilizaron dos cepas del antagonista para el control de Fusarium spp provenientes del laboratorio de Fitopatología de la FCA-UNC y del laboratorio de Fitopatología de la FCA-UNNE. El ensayo se realizó en el campo Mono Carayá (lote 124, Fracción A, Loreto, Misiones), en bloques al azar con tres tratamientos y tres repeticiones, siendo T1:(TR) UNNE, T2:(TR) UNC, T3: (TR) UNNE + (TR) UNC y T Testigo. Los biocontroladores se aplicaron de manera individual y combinados, a razón de $2.5 \mathrm{cc} /$ planta. Se cuantificó las variables incidencia I (PE/PS)*100y mortalidad post-plantación, siendo PE población enferma (número de individuos con presencia de sintomatología), y PS población sana (número de individuos con ausencia de sintomatología), respectivamente.

Resultados:Los datos obtenidos fueron los siguientes T1: $2 \%$, T2: $3 \%$, T3: 9\%, T4: 20\% de incidencia, y T1: 3\%, T2: 3\%. T3: 1\% y T4: 9\% de muerte post plantación.Se puede concluir provisoriamente, que los resultados demuestran un efecto positivo sobre el control de Fusarium spp. en los materiales tratados con Trichoderma spp logrando menor porcentaje de muerte post plantación y de incidenciade la enfermedad. 